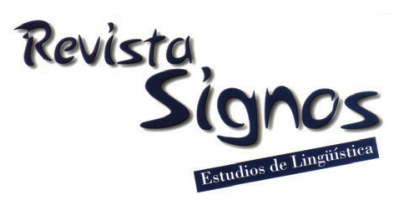

\title{
Una propuesta de taxonomía de marcadores metadiscursivos para el discurso académico- científico escrito en español
}

\author{
A taxonomy of metadiscourse in written scientific Spanish
}

\author{
Millaray Salas Valdebenito \\ Pontificia Universidad Católica de Valparaíso \\ Chile \\ millysalas@gmail.com, millaray.salas.v@mail.pucv.cl
}

Recibido: 13-I-2014 / Aceptado: 18-VIII-2014

\section{Resumen}

A partir del modelo reflexivo de metadiscurso (Ädel, 2006), el presente estudio propone una taxonomía de tres tipos de marcadores metadiscursivos que emergen de un estudio de corpus en el que se analizó manualmente 238 artículos de investigación científica (AICs) provenientes de tres disciplinas (Medicina, Economía y Lingüística). El primer tipo de metadiscurso corresponde al metadiscurso personal (MP), que queda conformado por aquellas expresiones lingüísticas en las que se hace mención explícita de los participantes del acto comunicativo, a saber, escritor, lector, o ambos. En el segundo tipo, el metadiscurso impersonal (MI), los participantes se mencionan de forma oblicua, utilizando estrategias de desagentivación y despersonalización. Por último, el metatexto (MT) corresponde a aquellas expresiones lingüísticas empleadas en forma reflexiva para referirse al propio texto mientras éste se despliega. En el MP, se identificaron tres categorías funcionales (auto-menciones, marcadores relacionales y referencia a los participantes), en el MI dos (auto-menciones impersonales y directivos impersonales) y en el MT tres (referencias al código, marcadores endofóricos y reformuladores explicativos). Uno de los aspectos más relevantes de esta taxonomía es que introduce tres categorías de marcadores metadiscursivos con realizaciones a nivel léxico-gramatical que son propias del sistema de la lengua española. El artículo discute algunas implicancias pedagógicas y aplicaciones de la taxonomía propuesta para los estudios basados en corpus.

Palabras Clave: Metadiscurso, taxonomía, modelo reflexivo, escritura académicacientífica, AICs, español. 


\begin{abstract}
Drawing on the reflexive model of metadiscourse (Ädel, 2006), the present study proposes a taxonomy of three types of metadiscourse markers, namely, Personal Metadiscourse (PM), Impersonal Metadiscourse (IM) and Metatext (MT). The proposed taxonomy is derived from a close manual analysis of 238 recent empirical research articles from three different disciplines (Medicine, Economics and Linguistics) from Spanish-medium journals indexed in Web of Science. PM encompasses linguistic expressions in which the participants of the communicative event (writer and/ or reader) are explicitly mentioned. IM comprises all references to the participants which are realized obliquely, by relying on an array of depersonalizing strategies. Last, MT groups all those expressions which refer to the text as it unfolds. In MP, three functional categories were identified (self mentions, relational markers and reference to participants), two in IM (impersonal self mentions and impersonal directives), and three categories in MT (references to the code, code glosses, and endophoric markers). One of the more significant findings to emerge from this study is that three MD categories, namely, self mentions realized by means of the verbal system (SMVS), impersonal discourse verb realizations, and impersonal directives (IDIR), are particular to the Spanish language. Pedagogical implications and applications of the taxonomy to corpus-based studies are discussed.
\end{abstract}

Key Words: Metadiscourse, taxonomy, reflexive model, scientific writing, RAs, Spanish.

\title{
INTRODUCCIÓN
}

Uno de los rasgos distintivos del lenguaje humano es su reflexividad (Hockett, 1960; Lyons, 1977). Hasta donde se sabe, todas las lenguas poseen expresiones para referirse, describir y comentar sobre el propio lenguaje. El estudio de esta característica central del lenguaje humano ha ocupado a un grupo importante de lingüistas desde ya hace más de tres décadas, quienes han propuesto diferentes modelos para aproximarse al objeto de estudio hoy conocido como metadiscurso, gracias a Harris (1959), quien acuñó el término.

El metadiscurso o reflexividad del discurso (Mauranen, 2010) es un rasgo de la prosa académica-científica que abarca tres aspectos (Ädel, 2006): 1) cómo los escritores científicos se refieren a sí mismos, 2) cómo se dirigen y se relacionan con su audiencia y 3) cómo se refieren a sus propios textos. El primero atañe al fenómeno de la auto-referencia o auto-menciones por parte del autor del texto. En relación a este punto, algunos estudios contrastivos de artículos de investigación científica (AICs de aquí en adelante) escritos en inglés y otras lenguas han encontrado que en algunas disciplinas los científicos tienden a emplear pronombres de primera persona (singular y plural), mientras que en otras se favorece un estilo más impersonal (Hyland, 2005; Fløttum, Kinn \& Dahl, 2006). El segundo aspecto está relacionado, por ejemplo, con 
la existencia de marcadores relacionales que señalan compromiso (e.g. 'consideremos la siguiente hipótesis', 'debe notarse que', etc.), a través de los cuales los autores explícitamente establecen una relación con el lector. Al respecto, se ha sugerido que los escritores científicos usan estratégicamente el imperativo en inglés con el propósito de comprometer a sus lectores (Swales, Ahmad, Chang, Chávez, Dressen \& Seymour, 1998). La tercera manifestación del metadiscurso en la escritura científica se realiza en el empleo de metatexto, el cual corresponde a "portions of text whose job is, not to give information about the subject proper but rather to clarify the structure of the text" (Enkvist, 1997: 200). Con respecto a este tercer aspecto, algunos estudios han descubierto que la prosa científica escrita en inglés suele ser más amistosa con el lector que su contraparte en otras lenguas, tales como el alemán (Siepmann, 2006), finés (Mauranen, 1993) o noruego (Fløttum et al., 2006). Esto quiere decir que los científicos angloparlantes tienden a allanar el camino a sus lectores a través de la inclusión de mecanismos léxico-gramaticales como reformuladores explicativos y marcadores endofóricos.

La línea de investigación que se enfoca en el estudio del metadiscurso ha tenido un gran desarrollo en las últimas décadas, entre lo que se cuenta la propuesta de varios modelos teóricos de estudio de este fenómeno y taxonomías para la búsqueda de expresiones metadiscursivas en textos orales y escritos (e.g. Williams, 1981; Vande Kopple, 1985; Crismore, 1989; Bunton, 1999; Dafouz-Milne, 2003; Hyland, 2005; Ifantidou, 2005; Ädel, 2006). Sin embargo, las categorías funcionales del metadiscurso que se han propuesto en la literatura toman como referente la lengua inglesa. Una revisión preliminar de la bibliografía disponible nos permite observar que no existe una taxonomía de marcadores metadiscursivos para el español, lo que plantea un problema para aquellos investigadores que trabajan con corpus de textos escritos en esta lengua. Al respecto, cabe hacer notar que, salvo escasas excepciones (FuertesOlivera, Velasco-Sacristán, Arribas-Baño \& Samaniego Fernández, 2001; DafouzMilne, 2003; Mur Dueñas, 2011), la mayoría de los estudios de metadiscurso en la prosa científica en español (Valero-Garcés, 1996; Moreno, 1997, 1998, 2004; Mur Dueñas, 2003, 2007; Beke, 2005; Cubo de Severino, 2005; Martínez, 2005; Müller, 2007; García Negroni, 2008; Carciu, 2009; Sheldon, 2009; Aguirre, 2010; Bolívar, Beke \& Shiro, 2010; Pérez-Llantada, 2010; Williams, 2010; Lorés-Sanz, 2011a, 2011b; Murillo, 2012) han adoptado la taxonomía de Hyland (2005), sin considerar fundamental el hecho de que fue propuesta para el inglés y no debería partirse del supuesto de que en español existen los mismos mecanismos metadiscursivos. Por ello, el objetivo de este trabajo es proponer una taxonomía de marcadores metadiscursivos para el discurso académico-científico escrito en español. Dicha taxonomía es el resultado de un análisis en el que se empleó un método ascendente-descendente. Es decir, no se partió el análisis con una taxonomía a priori de categorías estancas sino que se llegó a ella en base a la observación de datos lingüísticos auténticos, tras anotar manualmente 238 AICs escritos en español que conforman el Corpus MEL-2011 (para mayor detalle, ver sección 3.1). 
El artículo está organizado de la siguiente forma. En la próxima sección, se realiza primero una breve exposición de las dos grandes perspectivas desde las que se ha estudiado el fenómeno del metadiscurso: una integradora y otra no integradora. A continuación, y debido a que este estudio adopta el modelo de Ädel (2006), se analiza la propuesta de esta autora y se fundamenta la elección de este modelo.

En la tercera sección del trabajo, se exponen brevemente los hallazgos del estudio basado en corpus que informa la taxonomía propuesta, específicamente en cuanto a las categorías de marcadores metadiscursivos que emergieron de la investigación. En este acápite se presenta en detalle cada una de las ocho categorías funcionales que se relevaron del análisis manual del Corpus MEL-2011, las cuales se subdividen en dieciocho grupos de rasgos léxico-gramaticales que señalan el metadiscurso en textos científicos escritos en español. Se hace mención especial de las tres categorías que emergieron del estudio y que no aparecen en ninguna taxonomía previa, por tratarse de realizaciones que solo son posibles en el sistema léxico-gramatical del español y no en el del inglés.

En la conclusión, se resumen los aportes y limitaciones de la taxonomía propuesta. También se hacen algunas sugerencias en cuanto a su aplicación en el marco de los estudios basados en corpus y en la enseñanza de la escritura académica y científica.

\section{Marco de referencia}

\subsection{El estudio del metadiscurso desde dos perspectivas teóricas}

A riesgo de simplificar demasiado la complejidad de esta área de investigación, se podría decir que existen dos grandes tradiciones en el estudio del metadiscurso. De un lado, está aquella que usa una definición amplia y ve la interacción textual como el rasgo definitorio del metadiscurso. Por otro lado, está la que usa una definición acotada y pone a la reflexividad en el lugar central. La primera tradición ha sido etiquetada como 'integradora' (Mauranen, 1993) o 'modelo interactivo' (Ädel, 2005), mientras que la segunda ha sido denominada 'no integradora' (Mauranen, 1993) o ‘modelo reflexivo' (Ädel, 2005).

Una de las diferencias entre ambos enfoques tiene que ver con los fenómenos lingüísticos que se consideran propiamente metadiscursivos. La definición amplia de metadiscurso aglutina a los conectores, los mitigadores y enfatizadores, y la evaluación o postura. Ninguno de estos fenómenos se considera propiamente metadiscursivos desde la definición acotada. No obstante lo anterior, todos los modelos de metadiscurso que se mencionan aquí se inscriben dentro de un paradigma funcionalista del lenguaje. Sobre este punto, cabe aclarar que aquí estamos empleando el término funcionalista sensu lato, es decir, los modelos estudiados generalmente aluden a cómo el lenguaje funciona para que sus usuarios logren ciertos propósitos comunicativos (Hyland, 2005; Ädel, 2006). Sin embargo, el término funcionalista no siempre es empleado de la misma forma. Así, se puede decir que la mayoría de los modelos integradores y no 
integradores son funcionalistas en el sentido de que toman como supuesto la hipótesis metafuncional propuesta por Halliday (1970). A partir de ella, algunos investigadores proponen que el metadiscurso comprende tanto las funciones interpersonales como textuales (Vande Kopple, 1985; Crismore, 1989; Hyland, 2005), mientras que otros sostienen que el metadiscurso solo abarca la función textual (Mauranen, 2004).

En contraste, el modelo que hemos adoptado en esta investigación (Ädel, 2006) es funcionalista en un sentido diferente. En primer lugar, parte del modelo de Jakobson (1985) que propone seis funciones del lenguaje, entre las que se cuenta la función metalingüística o reflexiva. En segundo lugar, recurre a otro concepto funcionalista, el de reflexividad (Lyons, 1977), y le atribuye un rol central en deslindar lo que cuenta o no como metadiscursivo. Es por ello que se denomina el modelo reflexivo de metalenguaje.

\subsection{El modelo reflexivo de metadiscurso (Ädel, 2oo6)}

Ädel (2006) define metadiscurso como el texto acerca del texto en evolución, o el comentario explícito del escritor sobre su propio discurso mientras éste se despliega, lo cual deja en evidencia una consciencia sobre el texto actual o el uso del lenguaje per se y del escritor y lector actual en sus respectivos roles discursivos. Este concepto abarca dos dimensiones: el 'metatexto' y la 'interacción escritor-lector'. El primero alude a "reflexive linguistic expressions referring to the evolving text per se or its linguistic form" y el segundo a "references to the writer persona and the imagined reader qua writer and reader of the current text” (Ädel, 2005: 154).

Según esta autora, el metadiscurso exhibe cuatro propiedades características. En primer lugar, es una categoría difusa, un concepto cuya delimitación ha probado ser esquiva y dificultosa. Segundo, es una categoría funcional, en el sentido de que el énfasis está puesto en las funciones lingüísticas (Jakobson, 1985) que están asociadas con el material metadiscursivo, y no su supuesto (y polémico) contenido noproposicional. Tercero, las expresiones metadiscursivas pueden ser multifuncionales, esto es, pueden llevar a cabo simultáneamente varias funciones a nivel discursivo. Y, por último, el metadiscurso depende del contexto, es decir, para decidir si un mecanismo léxicogramatical está llevando a cabo una función metadiscursiva, el cotexto deber ser cuidadosamente inspeccionado.

El modelo reflexivo de metadiscurso introduce cuatro criterios para definir y acotar aquello que califica como metadiscurso:

1. Explicitud (basado en Mauranen, 1993): el requisito de que exista un comentario explícito e intencionado sobre el discurso.

2. Mundo del discurso: solo cuentan como metadiscursivas aquellas acciones que ocurren en el mundo discursivo (e.g. resaltar, presentar, poner de manifiesto) en vez de en el mundo real (e.g. llevar a cabo una investigación, entrevistar a participantes del estudio). 
3. E1 texto actual (basado en Mauranen, 1993): el metadiscurso hace referencia al texto actual más que a otros textos; lo segundo constituiría intertextualidad.

4. Hablante-escritor qua hablante-escritor y audiencia qua audiencia (en esto se excluye el metatexto): Una unidad lingüística solo cuenta como metadiscursiva si hace referencia al productor y al receptor del discurso en sus roles como participantes discursivos, es decir, en el mundo del discurso (en contraposición a ellos como personas reales en el mundo real).

Considerando los cuatro criterios mencionados arriba, la evaluación (o postura) queda excluida del ámbito del metadiscurso y se considera una categoría conceptualmente diferente: "Unlike metadiscourse, stance is not self-reflexive language; it does not involve the metalinguistic function" (Ädel, 2006: 40). De acuerdo a esta investigadora, en la evaluación hay tres funciones predominantes: la expresiva, la directiva y la referencial (orientada hacia las entidades en el mundo real). $\mathrm{Y}$ aunque existe un cierto traslape entre ambos conceptos (en ambos los participantes del discurso son componentes centrales), el metadiscurso conecta al escritor y al lector con el texto actual o con el mundo del discurso, mientras que la evaluación los conecta con el mundo real.

Ädel (2006) arguye que el empleo de la teoría de Roman Jakobson (1985) para delimitar las fronteras de este concepto presenta varias ventajas por sobre el modelo interactivo de metadiscurso (Crismore, 1989; Hyland, 2005). Entre ellas, 1) se enfatiza la reflexividad en este fenómeno discursivo, 2) se evita el problema de discutir el elemento proposicional y 3), se logra un concepto más situado, al incluir tanto al escritor como al lector.

En el modelo reflexivo se plantean dos variedades de metadiscurso: personal (MP) e impersonal (MI). Vale la pena aclarar que esta división no es teórica, sino que meramente intenta dar cuenta de dos realizaciones del metadiscurso a nivel de la superficie del texto. El MD hace referencia directa al escritor y/o lector del texto actual, ya sea a través de pronombres (i.e. yo, nosotros, Ud.) o sustantivos (e.g. el escritor, le lector). Esta referencia explícita a los participantes del acto comunicativo es en relación a su roles discursivos, como escritor o lector imaginado, respectivamente. Esto implica que, en cuanto al empleo del pronombre personal de primera persona singular, solo se consideran expresiones metadiscursivas personales aquellos tokens de este pronombre que se refieran al escritor presentándose a sí mismo como escritor, en vez de como un ser humano en el mundo real involucrado en otras actividades. Un criterio similar se adopta con las ocurrencias del pronombre personal de la primera persona plural. En contraste, el MI no hace referencia explícita al escritor o al lector, sino que se alude a estos participantes de forma oblicua. El MI se realiza a nivel léxico-gramatical en formas pasivas y variadas construcciones desagentivadas.

En cuanto a nuestra decisión de emplear el modelo reflexivo de metadiscurso (Ädel, 2006), existen al menos dos consideraciones relevantes. En primer lugar, se trata de un modelo que logra un alto grado de rigor teórico al circunscribir una categoría difusa 
como el metadiscurso, delimitando su alcance y deslindándola de otros fenómenos como la evaluación (o actitud), la mitigación, la intertextualidad y la atribución. En segundo lugar, al hacer énfasis en las propiedades reflexivas del metadiscurso, en vez de en su supuesto contenido no-proposicional, se evita una de las principales críticas y objeciones al concepto de metadiscurso (Mauranen, 1993; Sinclair, 2005). Varios autores han hecho ver que un mismo mecanismo metadiscursivo puede ser utilizado para llevar a cabo dos o más funciones simultáneamente: referencial y expresiva, metalingüística y directiva, etc. (Crismore, 1989; Mauranen, 2003; Ädel, 2005; Hyland, 2005; Ifantidou, 2005).

\section{Marco metodológico}

\subsection{Descripción del corpus y procedimiento}

En este estudio exploratorio-descriptivo del metadiscurso en la prosa científica escrita en español, se empleó un enfoque basado en corpus. Más específicamente, se adoptó un diseño tipo B (Biber \& Jones, 2009: 1298) en el que la unidad de análisis es cada texto individual y la meta es "examine differences between texts and text categories". Se compararon las frecuencias de ocurrencia de marcadores metadiscursivos entre los textos y los tres subcorpora. El estudio comprendió dos fases: una exploratoria en la que se analizó el 33\% de los textos con el fin de identificar categorías de expresiones metadiscursivas, y otra comprobatoria en la cual el resto de los textos (67\%) fueron analizadas con el propósito de poner a prueba la hipótesis de que existen diferencias estadísticamente significativas en el empleo de metadiscurso entre los tres subcorpora de AICs.

A pesar de que el estudio se basa en los criterios propuestos por Ädel (2006) para la delimitación de lo que cuenta como metadiscurso, se aplicó una metodología ascendente-descendente (top-down, bottom-up) en la fase exploratoria de la investigación. Es decir, no se partió de una taxonomía descrita en la literatura especializada con categorías predeterminadas de marcadores metadiscursivos, sino que ellas emergieron del proceso de anotación manual del Corpus MEL-2011.

El Corpus MEL-2011 está compuesto por 238 AICs escritos en español provenientes de tres disciplinas (Lingüística, Economía y Medicina), representativas de las tres ramas de la ciencia (Fløttum et al., 2006): las humanidades, las ciencias sociales y las ciencias naturales, respectivamente. Por conveniencia, se empleó una muestra incidental (Polgar \& Thomas, 2000). Todos los artículos recabados cumplen con los siguientes criterios externos (Biber, 2004) de tal forma de garantizar tertium comparationis (Connor, 2004: 292), esto es, "a common platform of comparison or shared similarity between texts":

1. Estar indexados en Thomson ISI.

2. Estar disponibles en formato digital (HTML o PDF). 
3. Estar escritos directamente en español (se excluyeron las traducciones).

4. Tener un solo autor.

5. Ser empíricos (se excluyeron ensayos, discusiones teóricas, revisiones de la literatura y propuestas metodológicas).

6. Haber sido publicados entre enero del 2005 y enero del 2010.

En cuanto a las características internas de nuestro corpus, se seleccionaron artículos completos, es decir, el texto completo, con todas sus secciones, exceptuando referencias bibliográficas, tablas y figuras. Ya que el número de palabras no quedó distribuido uniformemente en los tres subcorpora, se determinó la frecuencia relativa de los rasgos textuales estudiados. Dicho de otro modo, no se consideró la tasa bruta de ocurrencias de dichos marcadores sino que los datos fueron normalizados. La Tabla 1 muestra el detalle del corpus empleado en este estudio (ver Anexo 1 para revisar el listado completo de revistas indexadas de las que fueron recabados los artículos).

Tabla 1. Descripción del Corpus MEL-2011.

\begin{tabular}{|c|c|c|}
\hline CORPUS & TOTAL DE TEXTOS POR DISCIPLINA & TOTAL DE PALABRAS \\
\hline Lingüística & 93 & 706.529 \\
\hline Economía & 79 & 694.199 \\
\hline Medicina & 66 & 223.972 \\
\hline & TOTAL: 238 & TOTAL: 1.624 .700 \\
\hline
\end{tabular}

En la etapa exploratoria, se anotó manualmente el 33\% (N=85) de los AICs con el fin de identificar tokens de marcadores metadiscursivos. Se optó por el análisis manual, en vez de una búsqueda automática, debido a las propiedades inherentes del objeto de estudio, a saber, su naturaleza difusa, multifuncional y dependiente del contexto (ver sección 2.1 para más detalles). Esto significó que antes de decidir si una unidad léxico-gramatical estaba llevando a cabo una función metadiscursiva, se emplearon los criterios propuestos por Ädel (2006) expuestos arriba (ver sección 2.1) y se examinó detenidamente el co-texto. Como resultado de esta fase, tres categorías de MD, dos de MI y tres de MT emergieron de los datos. Estas categorías se emplearon como ítemes de búsqueda en la etapa comprobatoria, en la cual el resto de los artículos (67\%) también fueron anotados.

En relación al método para contar unidades metadiscursivas, se siguió el método descrito por Ädel (2006) que consiste en contar cada unidad lingüística (e.g. pronombre, verbo, frase endofórica, etc.) dentro de una cláusula u oración como una ocurrencia distinta de metadiscurso. Como ejemplo, en la oración 'A continuación describimos los datos utilizados en este trabajo', se cuentan tres unidades metadiscursivas: 'a continuación' (marcador endofórico), 'describimos' (auto-mención realizada a través del sistema verbal usando una desinencia verbal de primera persona plural) y 'este trabajo' (referencia al texto/código). 


\section{La taxonomía propuesta}

A continuación, se presenta la taxonomía de marcadores metadiscursivos propuesta para el discurso académico-científico escrito en español. Dentro de la taxonomía, se hace una primera distinción entre Metadiscurso Personal (MP), Metadiscurso Impersonal (MI) y Metatexto (MT). El MP agrupa a todas aquellas expresiones metadiscursivas que aluden explícitamente a los participantes del evento comunicativo (escritor y/o lector). Por su parte, el MI abarca las expresiones metadiscursivas en las que se alude a dichos participantes en forma oblicua o indirecta. Por último, el MT incluye las expresiones metadiscursivas que no están orientadas a la interacción entre escritor-lector, sino que hacia el código o discurso mismo.

La taxonomía está compuesta por ocho grandes categorías (o categorías agregadas) de marcadores metadiscursivos (ver Tabla 2). En el MP, se identificaron tres categorías (auto-menciones, marcadores relacionales y referencia a los participantes), en el MI dos (auto-menciones impersonales y directivos impersonales) y en el MT tres (referencias al código, marcadores endofóricos y reformuladores explicativos). Cada una de estas categorías cuenta con una etiqueta funcional que intenta dar cuenta del acto metadiscursivo que este grupo de marcadores léxico-gramaticales lleva a cabo en su contexto discursivo inmediato (Ädel, 2006).

Como se observa en la Tabla 2, las tres categorías funcionales de MP y las dos de MI se desagregan o subdividen a su vez en categorías más pequeñas. La mayor parte de estas categorías desagregadas toman una etiqueta funcional, pero cuatro de ellas adoptan una etiqueta morfosintáctica (e.g. VDSI, Verbos discursivos Se Impersonal) y otras cuatro una híbrida, es decir, funcional-morfosintáctica (e.g. AMSPSG Auto Menciones realizadas a través del Sistema Pronominal en forma Singular). El etiquetaje propuesto puede necesitar revisión, pero debe considerarse como un primer intento por crear una taxonomía para el discurso científico escrito para la lengua española. 
Tabla 2. Propuesta de taxonomía de marcadores metadiscursivos para el discurso académico-científico escrito en español.

\begin{tabular}{|c|c|c|}
\hline METADISCURSO PERSONAL (MP) & $\begin{array}{l}\text { METADISCURSO } \\
\text { IMPERSONAL (MI) }\end{array}$ & METATEXTO (MT) \\
\hline $\begin{array}{l}\text { 1. AUTO-MENCIONES (AM) } \\
\text { AMSP: Auto menciones realizadas por medio } \\
\text { del sistema pronominal } \\
\text { - AMSPSG (pronombres de primera } \\
\text { persona singular): yo, me, mi(s) } \\
\text { AMSPPL (pronombres de primera } \\
\text { persona plural): nosotros, nos, nuestro/ } \\
\text { a(s) } \\
\text { AMSV: Auto-menciones realizadas por } \\
\text { medio del sistema verbal empleando verbos } \\
\text { discursivos } \\
\text { AMSVSG (desinencias de primera } \\
\text { A persona singular) } \\
\text { AMSVPL (desinencias de primera } \\
\text { persona plural) }\end{array}$ & $\begin{array}{l}\text { 4. AUTO MENCIONES } \\
\text { EN FORMAS } \\
\text { IMPERSONALES } \\
\text { EMPLEANDO } \\
\text { VERBOS } \\
\text { DISCURSIVOS (AMI) } \\
\text { - VDSI: Formas } \\
\quad \text { pasivas con "se } \\
\text { impersonal" } \\
\text { - VDVP: Formas } \\
\text { pasivas con verbo } \\
\text { ser/estar y sin } \\
\text { agente } \\
\text { VDP: Perífrasis } \\
\text { verbal } \\
\text { VDN: } \\
\text { Nominalizaciones }\end{array}$ & $\begin{array}{l}\text { 6. REFERENCIAS AL } \\
\text { CÓDIGO (REFC) } \\
\text { - } \quad \text { REFT: Referencias } \\
\text { al texto completo } \\
\text { - } \\
\text { REFPT: Referencias } \\
\text { a parte del texto } \\
\text { REFOMS: } \\
\text { Referencias a otros } \\
\text { modos semióticos }\end{array}$ \\
\hline $\begin{array}{l}\text { 2.MARCADORES RELACIONALES } \\
\text { (MREL) } \\
\text { DIRP: Directivos personales } \\
\text { NINC: "Nosotros" inclusivo }\end{array}$ & $\begin{array}{l}\text { 5. DIRECTIVOS } \\
\text { IMPERSONALES } \\
\text { (DIRI) }\end{array}$ & $\begin{array}{l}\text { 7. MARCADORES } \\
\text { ENDOFÓRICOS } \\
\text { (FOR) }\end{array}$ \\
\hline $\begin{array}{l}\text { 3. REFERENCIAS A LOS } \\
\text { PARTICIPANTES (REFP) } \\
\text { REFA: Referencia al autor } \\
\text { REFL: Referencia al lector }\end{array}$ & & $\begin{array}{l}8 . \\
\text { REFORMULADORES } \\
\text { EXPLICATIVOS (REF) }\end{array}$ \\
\hline
\end{tabular}

\subsection{Marcadores de metadiscurso personal (MP)}

Tres grandes categorías funcionales de MP emergieron del estudio, a saber, 1) auto menciones (AM), 2) marcadores relacionales (MREL) y 3) referencia a los participantes (REFP).

\subsubsection{Auto menciones (AM)}

Esta categoría se desagrega en dos subcategorías: AMSP o auto menciones realizadas por medio del sistema pronominal (i.e. pronombres de primera persona singular y plural) y AMSV o auto menciones realizadas a través del sistema verbal. Ya que el español es una lengua pro-drop, en esta segunda realización el pronombre personal se elide y la auto-mención se instancia en la desinencia verbal. Para la categoría desagregada AMSV, solo se consideraron verbos discursivos (Hyland, 2000). En este estudio, se utiliza este término paraguas en un sentido bastante amplio, ya que estamos incluyendo todas las ocurrencias en el corpus de desinencias verbales de primera persona singular y plural de los siguientes tipos de verbos: 
- Verba dicendi (e.g. denominar, afirmar, decir, señalar, enunciar, etc.)

- Verbos de percepción visual (Müller, 2007) (e.g. ver, notar, observar, advertir, etc.)

- Verbos de investigación (Fløttum et al., 2006) (e.g. analizar, asumir, considerar, examinar, encontrar, estudiar, etc.)

- Verbos que se refieren a procesos que involucran una representación verbal o gráfica (Hyland, 2000) (e.g. describir, ilustrar, ejemplificar, presentar, exponer, resumir, etc.)

- Verbos que que se refieren a los procesos de estructurar el texto y guiar al lector (Hyland, 2000) (e.g. comenzar con, enfocarse en, centrarse en, explorar o abordar (un problema o temática), regresar (sobre este punto), etc.).

- Verbos de posicionamiento (Fløttum et al., 2006) o verbos persuasivos (Biber, 1988) (e.g. postular, aseverar, sostener, argumentar, plantear, argüir, rechazar, objetar, hacer hincapié, recalcar, etc.)

- Verbos de cognición (Hyland, 2002), verbos privados (Biber, 1988) (e.g. creer, pensar, asumir, interpretar, analizar, concluir, hipotetizar, etc.).

La lista completa de verbos discursivos que emergieron de la anotación manual de los textos se encuentra en el Anexo 2.

\subsubsection{Marcadores relacionales (MREL)}

Esta categoría se desagrega en dos subcategorías: directivos personales (DIRP) y el empleo del nosotros inclusivo (NINC). Los directivos personales (DIRP) corresponden a formas imperativas de primera persona plural (e.g. analicemos, consideremos, supongamos, observemos, recordemos, tomemos como ejemplo, pongamos como ejemplo, veamos un ejemplo, no olvidemos, pensemos, etc.). La segunda categoría desagregada corresponde al empleo de verbos con desinencia de primera persona plural que tienen carácter inclusivo, es decir, que son usados por el escritor para comprometer al lector (e.g. en el ejemplo que acabamos de ver, tenemos, observamos, comparamos, logramos, etc.). En muchos casos, estos verbos son parte de una cláusula condicional y van antecedidos de la conjunción 'si' (e.g. si agregamos... podemos...; si las comparamos, hacemos...; si relacionamos..., observamos..., etc.). En estos casos, el acto de habla que el productor del texto busca realizar es que el lector imagine escenarios hipotéticos (Ädel, 2006). En este caso hemos escogido la abreviatura NINC (Nosotros Inclusivo) para referirnos a estos verbos terminados en primera persona plural que son usados por el autor con una función asociativa (Montero-Fleta, Montesinos, Pérez \& Turney, 2004). 


\subsubsection{Referencia a los participantes (REFP)}

Esta tercera categoría está conformada por todas las referencias a los participantes del acto comunicativo. La categoría Referencias al Autor (REFA) comprende todas las alusiones al productor del texto (e.g. el autor agradece..., traducción del autor, las traducciones son de la autora, el autor es investigador responsable,... son responsabilidad exclusiva del autor, ... se pueden solicitar al autor, la versión en inglés puede ser obtenida del autor, el autor de este ensayo / artículo, etc.). Por otra parte, la categoría Referencias al Lector (REFL) abarca todas las alusiones al receptor del discurso (e.g. recomendamos al lector interesado visitar..., los lectores interesados en pormenores de la metodología..., a los que quieren hacerse una idea aproximada de... les refiero a la bibliografía de..., etc.).

A continuación, el Gráfico 1 muestra la distribución de los tres tipos de marcadores de MP (Auto Menciones -AM-, Marcadores Relacionales -MREL- y Referencias a los Participantes -REFP-), y sus correspondientes categorías desagregadas, en los tres subcorpora. Como se observa, existen diferencias estadísticamente significativas entre el corpus de Lingüística y el corpus de Economía ( $p=0,0008245)$ y Medicina $(p=0,001021)$ en términos de frecuencias relativas del conjunto de marcadores de MP. Los lingüistas son los que despliegan más mecanismos de MP en sus textos $(4,94$ cada 1000 palabras), en contraste con los economistas (2,94) y los médicos $(3,04)$. También se puede apreciar que de los tres tipos de MP, los más empleados son las auto menciones.

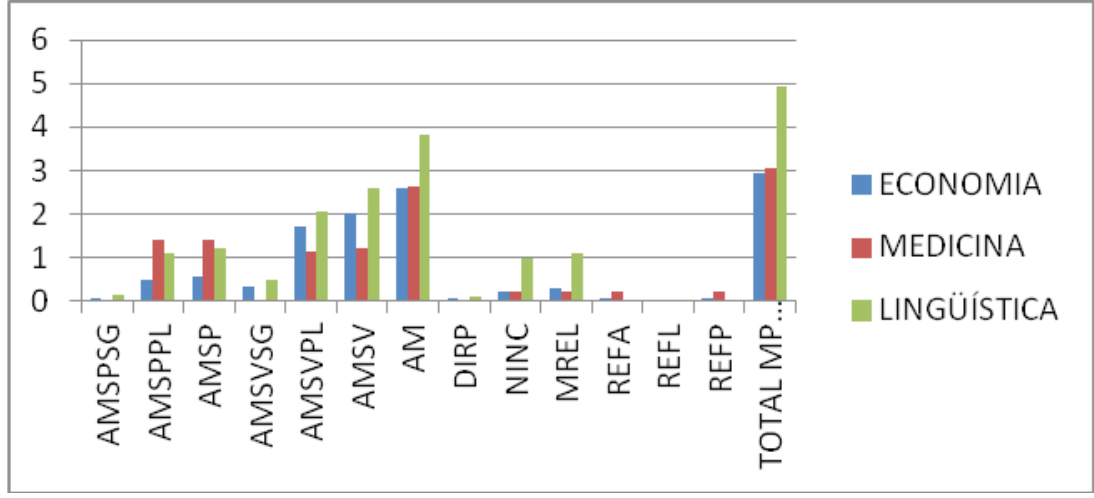

Gráfico 1. Frecuencias de ocurrencia de mecanismos de MP en las tres disciplinas del Corpus MEL-2011. 


\subsection{Marcadores de metadiscurso impersonal (MI)}

Dos grandes categorías funcionales de MI emergieron del estudio, a saber, 1) auto menciones a través de formas impersonales de verbos discursivos (VDI) y 2) directivos impersonales (DIRI).

3.2.1. Automenciones a través de formas impersonales de verbos discursivos (VDI)

Esta categoría se desagrega en cuatro subcategorías, correspondientes a cuatro realizaciones impersonales: (1) pasivas con 'se impersonal' (VDSI), (2) pasivas con 'ser' sin agente (VDVP), (3) perífrasis verbal (VDP) y (4) nominalización (VDN). Abajo, presentamos algunos ejemplos del se impersonal (i.e. pasivas con 'se') empleado como marcador de MI:

En el apartado siguiente se describe el modelo econométrico utilizado y el método para descomponer el diferencial salarial (ECO REA XV(43)).

Finalmente, se debe recalcar que la ansiedad registrada por el instrumento utilizado puede variar en el tiempo, y que los tipos de ansiedad pueden variar (MED GMM 142(3)).

Estas dos definiciones evidencian que el concepto de metadiscurso puede variar según el aspecto que se quiere enfatizar, tal como se señaló en la introducción a este artículo (LING SIGNOS 38(57)).

A continuación, ilustramos el empleo de pasivas con 'ser' sin agente (VDVP) como marcador de MI con tres ejemplos sacados del corpus, uno de cada disciplina:

En contraste, la voz médica (.32) y en especial la voz educativa (.68) presentan una alta proporción de uso del tiempo presente (ejemplos 4 y 5), que hace más inmediata y latente la connotación de objetividad experiencial del uso del modo indicativo ya mencionado para estas voces sin marcación de cortesía (LING EEFF 41).

Según la hipótesis planteada, el modelo 2 debe ser significativamente más predictivo y explicativo de la inviabilidad de las explotaciones agrícolas (ECO REA XV(44)).

Los resultados a corto plazo expuestos en esta revisión permiten ser optimistas respecto a la artroplastia como método de tratamiento de determinados casos de artrosis y artritis de tobillo (MED RECOT $52(4))$.

La tercera forma léxico-gramatical que emplean estos escritores para marcar el Metadiscurso Impersonal es la perífrasis verbal (VDP) en la que el verbo principal es un verbum dicendi u otro verbo discursivo. Veamos algunos ejemplos extraídos del corpus: 
Para discutir en qué sentido se entiende aquí el carácter "mediatizado" del discurso público mapuche, conviene precisar que se ha preferido este término por sobre el de "mediado" sólo para evitar la confusión que se da con cierta frecuencia en nuestro medio de atribuir el carácter mediador exclusivamente a los medios de prensa de comunicación masiva (LING EEFF 40).

Puede argumentarse, por tanto, que la productividad depende del tamaño de la explotación. No hay ninguna razón para pensar que las dos variables dicotómicas expresando la especialización productiva de la explotación sean endógenas (ECO REA XV (44)).

Es importante recalcar el hecho de que las medidas de PA se han realizado en épocas de temperatura ambiente similar, pues es bien conocido que en verano, debido al calor ambiental, la PAS puede descender hasta $12 \mathrm{mmHg}$ y la PAD hasta $5 \mathrm{mmHg}$ respecto al invierno, con el consiguiente efecto sobre las tasas de control (MED H 22(1)).

La última realización de VDI empleando verbos discursivos corresponde a las nominalizaciones (VDN), que abajo ilustramos con tres ejemplos de nuestro corpus:

En los casos en que el análisis no presenta ninguna complicación me limito a la exposición del mismo (LING SIGNOS 39 (61)).

Mención especial se debe hacer sobre la cohorte hipotética de la figura 2, con las limitaciones referidas, sintetiza los indicadores antes mencionados y sus efectos en el egreso de la licenciatura y el ingreso a la especialización (MED GMM 141(2)).

En el apartado 2 de este trabajo sobre Fuentes estadísticas, se presenta una explicación más detallada sobre esta cuestión (ECO REA XIII (37)).

\subsubsection{Directivos impersonales (DIRI)}

Los directivos impersonales se consideran un subtipo de marcadores relacionales (Hyland, 2005), pero a diferencia de las dos categorías de marcadores relacionales que ya mencionamos (DIRP y NINC), con los Directivos Impersonales el escritor le indica o sugiere al lector un curso de acción a seguir. En el caso particular de nuestro estudio, cuando empleamos este término nos referimos a una de las tres realizaciones siguientes:

1. Verbos en modo imperativo que emplean el 'se impersonal', (e.g. véase, obsérvese, considérese, nótese, recuérdese, téngase en cuenta, supóngase, adviértase, añádase, compárese, consúltese, piénsese, etc.).

2. Otras formas verbales, incluyendo verbos modales y perífrasis verbales, que implican una orden o sugerencia (e.g. consultar, observar, recordar, se puede ver, se puede consultar, puede verse, puede encontrarse, sería bueno ver, debe recordarse, etc.). 
3. La abreviatura de una forma imperativa en latín que significa confróntese o compárese (e.g. Cfr., cf.), ver o véase (e.g. Vid., V., v.).

En el Gráfico 2 se muestran las frecuencias relativas (cada 1.000 palabras) de los dos tipos de marcadores de MI (Auto menciones en formas impersonales empleando verbos discursivos -AMI- y Directivos Impersonales -DIRI), y en el caso de AMI, de sus categorías desagregadas. Al igual que en el caso del MP, existen diferencias estadísticamente significativas entre el corpus de Lingüística y el corpus de Economía $(\mathrm{p}=0,003921)$ y Medicina $(\mathrm{p}=0,008411)$, pero no entre Economía y Medicina ( $\mathrm{p}$ $=0,6441)$ en términos de frecuencias relativas del conjunto de marcadores de MI. Los lingüistas son los que despliegan más mecanismos de MI en sus textos (7,05 cada 1000 palabras), en contraste con los economistas $(4,76)$ y los médicos $(4,68)$. También se puede apreciar que existen diferencias significativas entre el empleo de las AMI y los DIRI en las tres disciplinas. Los lingüistas y los economistas emplean más marcadores de ambos tipos que sus contrapartes en el campo de la medicina.

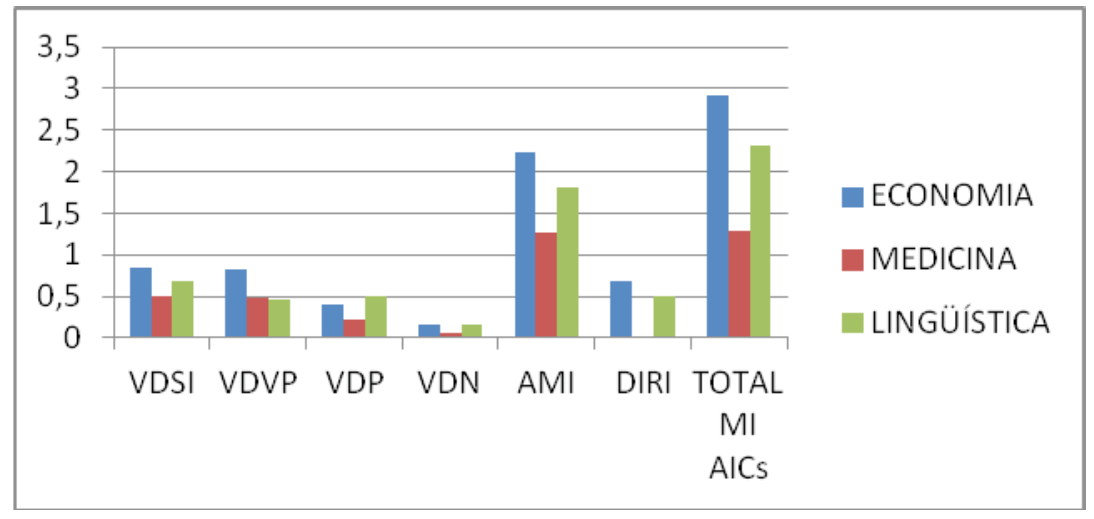

Gráfico 2. Frecuencias de ocurrencia de mecanismos de MI en las tres disciplinas del Corpus MEL-2011.

\subsection{Marcadores de metatexto (MT)}

Tres grandes categorías funcionales de MT emergieron del estudio, a saber, 1) referencias al código (REFC), 2) marcadores endofóricos (FOR) y 3) reformuladores explicativos (REF).

\subsubsection{Referencias al código (REFC)}

En relación a la primera categoría, encontramos tres tipos de referencias al código: referencias al texto (REFT), referencias a partes del texto (REFPT) y referencias a otros modos semióticos, específicamente imágenes (REFPV). Algunos ejemplos de marcadores de referencias al texto (REFT) son 'trabajo', 'artículo', 'ensayo' y 'comunicación'. Por referencias a partes del texto (REFPT), nos referimos a cualquier 
mención que haga el autor de una sección del texto, por ejemplo, al hablar de acápite, apartado, epígrafe o al mencionar el nombre de una sección del texto, tal como el Anexo, la sección de resultados, o los pies de página. Por último, las referencias a otros modos semióticos (REFOMS) corresponden a las menciones por parte del autor de las imágenes que incluye el texto y que incluyen tablas, gráficos y figuras.

\subsubsection{Marcadores endofóricos (FOR)}

La cuarta categoría corresponde a los Marcadores Endofóricos (FOR). Hyland (1998) define los marcadores endofóricos como aquellas expresiones lingüísticas que tienen la función de apuntar o señalar, en forma anafórica o catafórica, a otras porciones de un texto que se está leyendo o escribiendo. De esta forma, pueden ser comparados a señales de tránsito en el sentido de que ayudan a los lectores a navegar a través del texto (Ädel, 2006). En el Anexo 3, el lector encontrará una lista exhaustiva de todos los marcadores endofóricos que encontramos en el Corpus MEL-2011 y que están divididos en cuatro grupos: 1) anafóricos, si apuntan hacia atrás en el texto, 2) catafóricos, si apuntan hacia adelante en el texto, 3) un grupo de marcadores endofóricos que podríamos decir que funcionan como deícticos (por el hecho de apuntar a lo que se está diciendo en ese preciso lugar / momento del texto escrito) y 4) un último grupo de marcadores endofóricos que no califican en ninguna de las categorías anteriores.

\subsubsection{Reformuladores explicativos (REF)}

Los reformuladores explicativos también se conocen como glosas de código o comentario al código en la literatura especializada sobre el metadiscurso (Vande Kopple, 1985; Cubo de Severino, 2005; Hyland, 2005; Ädel, 2006). Vande Kopple (1985) define las glosas de código como aquellas expresiones lingüísticas que ayudan a que el lector comprenda e interprete los significados de las palabras, frases y expresiones idiomáticas o el sentido particular de un determinado elemento en el texto (e.g. Renacimiento significa literalmente 'nuevo nacimiento'). Estos mecanismos metadiscursivos reformulan, explican, definen, o clarifican el sentido de un término. Ver Anexo 3 para revisar la lista completa de marcadores de reformulación que encontramos en nuestro corpus.

El Gráfico 3 muestra las frecuencias relativas (cada 1.000 palabras) en los tres grupos de AICs de los tres tipos de marcadores de metatexto (MT), a saber, Referencias al Código -REFC-, Reformuladores Explicativos -REF- y Marcadores Endofóricos -FOR-. Como se observa, los economistas emplean significativamente más marcadores metatextuales $(5,79)$ que sus contrapartes en las otras disciplinas (4,35 en lingüística y 3,42 en medicina). También puede observarse que de los tres mecanismos metatextuales, el que exhibe mayor en las tres disciplinas es el de Referencias al Código (REFC). 


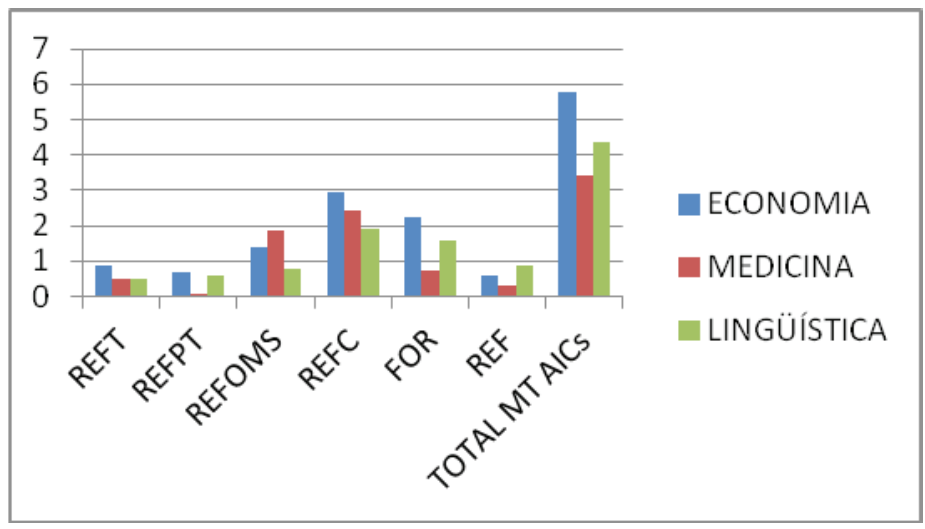

Gráfico 3. Frecuencias de ocurrencia de mecanismos de MT en las tres disciplinas del Corpus MEL-2011.

\section{CONCLUSIONES}

A diferencia de Ädel (2006), en cuyos criterios para delimitar lo que cuenta como metadiscurso se basa este estudio para proponer esta taxonomía, aquí se propone tres manifestaciones del metadiscurso para el español (en vez de solo dos): MP y MI en relación a la dimensión 'interacción escritor-lector' y MT para la dimensión 'metatexto'. Creemos necesario hacer la distinción entre MI y MT ya que el sistema de la lengua española permite varias realizaciones desagentivadas de auto menciones (VDSI, VDVP, VDP y VDN) y marcadores relacionales (DIRI) que no existen o no han sido descritas con suficiente detalle en las taxonomías sugeridas para el inglés (Vande Kopple, 1985; Crismore, 1989; Hyland, 2005; Ädel, 2006). Además, la existencia de esta variada gama de formas despersonalizadas de auto menciones en español ya ha sido reportado por otros investigadores (García Negroni, 2008). Por otra parte, la categoría MT debe ser deslindada de la de MI, ya que las expresiones metatextuales no aluden a la dimensión "interacción escritor-lector" sino que son empleadas por el autor para referirse al texto mientras se va desplegando.

De la anotación manual del Corpus MEL-2011 emergieron ocho categorías funcionales, tres de MP, dos de MI y tres de MT. Los marcadores metadiscursivos que quedan agrupados bajo el rótulo MP son de tres tipos: auto menciones, marcadores relacionales y referencia a los participantes. Dentro del MI, se relevaron dos categorías, a saber, auto menciones desagentivadas y directivos impersonales. Por último, se identificaron tres categorías de MT: referencias al código, marcadores endofóricos y reformuladores explicativos. Estas ocho categorías de expresiones metadiscursivas se desagregan en dieciocho, considerando la gama de realizaciones a nivel léxicogramatical que son posibles en la lengua española en el registro científico escrito. 
Uno de los hallazgos más interesantes del estudio que informa esta taxonomía es que de las dieciocho categorías desagregadas de MD que se proponen, tres no han sido previamente descritas en la literatura, probablemente por ser propias del español: (1) auto menciones realizadas a través del sistema verbal (AMSV) con el pronombre elidido, (2) auto menciones a través de varias formas impersonales de verbos discursivos (VDI) y (3) directivos impersonales (DIRI). Hasta donde hemos podido averiguar en nuestra indagación, este resultado no ha sido reportado anteriormente, al menos no desde un estudio cuantitativo basado en corpus. Estos resultados mejoran nuestra comprensión de cómo los diferentes sistemas lingüísticos posibilitan o constriñen las opciones retóricas de los escritores científicos que escriben en diferentes lenguas.

En cuanto a las implicancias pedagógicas de contar con una taxonomía de rasgos metadiscursivos para el español académico y científico escrito, se cuentan la posibilidad de modelar el uso apropiado de estos recursos a nivel de pregrado y postgrado según las normas y convenciones de cada comunidad disciplinar. El desconocimiento sobre cómo emplear el metadiscurso en forma eficaz puede convertirse en un obstáculo para el proceso de alfabetización científica y eventual inserción en la comunidad científica. Otra potencial aplicación de esta taxonomía es en la redacción de manuales de escritura académica-científica orientada a escritores novatos. Los autores de este tipo de textos pueden beneficiarse del inventario de expresiones metatextuales que aquí se presenta.

Dentro del ámbito de los estudios basados en corpus, el inventario de rasgos metadiscursivos que aquí se expone representa un aporte en el sentido de que posibilita la interrogación semi-automática de grandes corpora de textos académicos y científicos escritos en español. Como ya se ha mencionado, una búsqueda completamente automatizada de unidades metadiscursivas no es viable por la naturaleza del fenómeno. Aún así, el contar con una taxonomía y un listado exhaustivo de tokens de las diferentes categorías que la componen tiene el potencial de aumentar nuestra capacidad de hacer generalizaciones y de agilizar el análisis de grandes volúmenes de textos. 


\section{REFERENCIAS BILIOGRÁFICAS}

Ädel, A. (2005). On the boundaries between evaluation and metadiscourse. En E. Tognini-Bonelli \& G. Del Lungo Camiciotti (Eds.), Strategies in academic discourse (pp. 153-162). Ámsterdam: John Benjamins.

Ädel, A. (2006). Metadiscourse in L1 and L2 English. Ámsterdam: John Benjamins.

Aguirre, L. A. (2010). La construcción de la intersubjetividad en los informes de práctica profesional de Ingeniería. En V. M. Castel \& L. Cubo de Severino (Eds.), La renovación de la palabra en el bicentenario de la Argentina. Los colores de la mirada lingüistica (pp. 47-54). Mendoza: Editorial FFyL, UNCuyo.

Beke, R. (2005). El metadiscurso interpersonal en artículos de investigación. Revista Signos. Estudios de Lingüistica, 38(57), 7-18.

Biber, D. (1988). Variation across speech and writing. Cambridge: Cambridge University Press.

Biber, D. (2004). Representativeness in corpus design. En G. Sampson \& D. McCarthy (Eds.), Corpus Linguistics: Readings in a widening discipline (pp. 174-197). Londres: Continuum.

Biber, D. \& Jones, J. K. (2009). Quantitative methods in corpus linguistics. En A. Lüdeling \& M. Kytö (Eds.), Corpus Linguistics: An international bandbook (pp. 1286-1304). Berlín: Mouton de Gruyter.

Bolívar, A., Beke, R. \& Shiro, M. (2010). Las marcas lingüísticas del posicionamiento en las disciplinas: Estructura, voces y perspectivas discursivas. En G. Parodi (Ed.), Alfabetización académica y profesional en el siglo XXI: Leer y escribir desde las disciplinas (pp. 95-125). Santiago: Ariel.

Bunton, D. (1999). The use of higher level metatext in PhD theses. English for Specific Purposes, 18, S41-S56.

Carciu, O. (2009). An intercultural study of first-person plural references in biomedical writing. Ibérica, 18, 71-92.

Connor, U. (2004). Intercultural rhetoric research: Beyond texts. Journal of English for Academic Purposes, 3, 291-304.

Crismore, A. (1989). Talking with readers: Metadiscourse as a rhetorical act. Nueva York: Peter Lang.

Cubo de Severino, L. (2005). Representación mental del modelo de situación comunicativa en la lectura de manuales universitarios y artículos de investigación. Signo y Seña, 14, 141-157. 
Dafouz-Milne, E. (2003). Metadiscourse revisited: A contrastive study of persuasive writing in professional discourse. Estudios Ingleses de la Universidad Complutense, 11, 29-52.

Enkvist, N. E. (1997). Why we need contrastive rhetoric. Alternation, 4, 188-206.

Fløttum, K., Kinn, T. \& Dahl, T. (2006). Academic voices. Ámsterdam: John Benjamins.

Fuertes-Olivera, P., Velasco-Sacristán, M., Arribas-Baño, A. \& Samaniego Fernández, E. (2001). Persuasion and advertising English: Metadiscourse in slogans and headlines. Journal of Pragmatics, 33, 1291-1307.

García Negroni, M. M. (2008). Subjetividad y discurso científico-académico. Acerca de algunas manifestaciones de la subjetividad en el artículo de investigación en español. Revista Signos. Estudios de Lingüística, 41(66), 5-31.

Halliday, M.A.K. (1970). Language structure and language function. En J. Webster (Ed.), On Grammar (pp.173-195). Londres: Continuum.

Harris, Z. (1959). The Transformational Model of Language Structure. Antbropological Linguistics, 1(1), 27-29.

Hockett, Ch. (1960). Logical Considerations in the Study of Animal Communication. En W. E. Lanyon \& W. Tavolga (Eds.), Animal Sounds and Communication. (pp. 392-430). Washington, DC: American Institute of Biological Sciences.

Hyland, K. (1998). Hedging in scientific research articles. Ámsterdam: John Benjamins.

Hyland, K. (2000). Disciplinary discourses: Social interactions in academic writing. Londres: Longman.

Hyland, K. (2002). Activity and evaluation: Reporting practices in academic writing. En J. Flowerdew (Ed.), Academic discourse (pp. 115-130). Londres: Longman.

Hyland, K. (2005). Metadiscourse: Exploring interaction in writing. Londres: Continuum.

Ifantidou, E. (2005). The semantics and pragmatics of metadiscourse. Journal of Pragmatics, 37(9), 1325-1353.

Jakobson, R. (1985). Lingüistica y poética. Madrid: Cátedra.

Lorés-Sanz, R. (2011a). The study of authorial voice: Using a Spanish-English corpus to explore linguistic transference. Corpora 6(1), 1-24.

Lorés-Sanz, R. (2011b). The construction of the author's voice in academic writing: The interplay of cultural and disciplinary factors. Text and Talk, 31(2), 173-193. 
Lyons, J. (1977). Semantics. Cambridge: Cambridge University Press.

Martínez, I. (2005). Native and non-native writers' use of first person pronouns in the different sections of biology research articles in English. Journal of Second Language Writing 14(3), 174-190.

Mauranen, A. (1993). Cultural differences in academic rhetoric: A textlinguistic study. Frankfurt: Peter Lang.

Mauranen, A. (2004). Contrastive ESP rhetoric: Metatext in Finnish-English economics texts. En G. M. Saracino (Ed.), Writing for scholarly publication in English: Issues for nonnative speakers (pp. 126-156). San Cesario di Lecce: Manni Editori.

Mauranen, A. (2010). Discourse Reflexivity -A Discourse Universal? The Case of ELF. Nordic Journal of English Studies, 9(2), 13-40.

Montero-Fleta, B., Montesinos, A., Pérez, C. \& Turney, E. (2004). The reader and the writer in technical texts in English, Spanish and Catalan. Revista Española de Lingüística Aplicada, 16, 155-164.

Moreno, A. (1997). Genre constraints across languages: Causal metatext in Spanish and English RAs. English for Specific Purposes, 16(3), 161-179.

Moreno, A. (1998). The explicit signalling of premise-conclusion sequences in research articles: A contrastive framework. Text, 18(4), 545-585.

Moreno, A. (2004). Retrospective labelling in premise-conclusion metatext: An English-Spanish contrastive study of research articles on business and economics. Journal of English for Academic Purposes, Special Issue on Contrastive Rhetoric, 3(4), 321-340.

Müller, G. (2007). Metadiscurso y perspectiva: Funciones metadiscursivas de los modificadores de modalidad introducidos por ‘como’ en el discurso científico. Revista Signos. Estudios de Lingüística, 40(64), 357-387.

Mur Dueñas, P. (2003). Analysing stance in American and Spanish Business Management RAs: The case of sentence initial retrospective labels. Journal of English Studies, 4, 137-154.

Mur Dueñas, P. (2007). I/We focus on...: A cross-cultural analysis of self-mentions in business management research articles. Journal of English for Academic Purposes, 6(2), 143-162.

Mur Dueñas, P. (2011). An intercultural analysis of metadiscourse features in research articles written in English and in Spanish. Journal of Pragmatics, 43, 3068-3079. 
Murillo, S. (2012). The use of reformulation markers in Business Management research articles: An intercultural analysis. International Journal of Corpus Linguistics, 17(1), 64-90.

Pérez-Llantada, C. (2010). The discourse functions of metadiscourse in published academic writing: Issues of culture and language. Nordic Journal of English Studies, 9(2), 41-68.

Polgar, S. \& Thomas, S. (2000). Introduction to research in the health sciences. Melbourne, Australia: Churchill Livingstone.

Sheldon, E. (2009). From one I to another: Discursive construction of selfrepresentation in English and Castilian Spanish research articles. English for Specific Purposes 28(4), 251-265.

Siepmann, D. (2006). Academic writing and culture: An overview of differences between English, French and German. META, 51(1), 131-150.

Sinclair, J. (2005). Language as a string of beads: Discourse and the M-word. En E. Tognini-Bonelli \& G. Del Lungo Camiciotti (Eds.), Strategies in academic discourse (pp. 163-168). Ámsterdam: John Benjamins.

Swales, J. M., Ahmad, U. K., Chang, Y-Y., Chávez, D., Dressen, D. F. \& Seymour, R. (1998). Consider this: The role of imperatives in scholarly writing. Applied Linguistics, 19(1), 97-121.

Valero-Garcés, C. (1996). Contrastive ESP rhetoric: Metatext in Spanish-English Economics Texts. English for Specific Purposes, 15(4), 279-294.

Vande Kopple, W. (1985). Some exploratory discourse on metadiscourse. College Composition and Communication, 36, 82-93.

Williams, J. M. (1981). Style: Ten lessons in clarity and grace. Glenview, Illinois: Scott, Foresman and Co.

Williams, I. A. (2010). Cultural differences in academic discourse: Evidence from first-person verb use in the methods sections of medical research articles. International Journal of Corpus Linguistics, 15(2), 214-240. 


\section{ANEXOS}

Anexo 1: Listado de revistas científicas consultadas en la conformación del Corpus-MEL 2011.

\begin{tabular}{|c|c|c|}
\hline CÓDIGO & REVISTA & DIRECCIÓN ELECTRÓNICA \\
\hline RCC & Revista Chilena de Cirugía & $\begin{array}{l}\text { http://www.scielo.cl/scielo.php?script=sci_ } \\
\text { serial\&pid=0718-4026 }\end{array}$ \\
\hline GMM & Gaceta Médica México & $\begin{array}{l}\text { http://www.scielo.org.mx/scielo.php?script=sci_ } \\
\text { serial\&pid=0016-3813 }\end{array}$ \\
\hline RECOT & $\begin{array}{l}\text { Revista Española de Cirugía } \\
\text { Ortopédica y Traumatología }\end{array}$ & $\begin{array}{l}\text { http://www.sciencedirect.com/science/ } \\
\text { journal/18884415 }\end{array}$ \\
\hline RCE & Revista Clínica Española & $\begin{array}{l}\text { http://www.sciencedirect.com/science/ } \\
\text { journal/00142565 }\end{array}$ \\
\hline RMC & Revista Médica Chile & $\begin{array}{l}\text { http://www.scielo.cl/scielo.php?script=sci_ } \\
\text { serial\&pid=0034-9887 }\end{array}$ \\
\hline RCI & Revista Chilena de Infectología & $\begin{array}{l}\text { http://www.scielo.cl/scielo.php?script=sci_ } \\
\text { serial\&pid=0716-1018\&lng=es\&nrm=iso }\end{array}$ \\
\hline $\mathrm{H}$ & $\begin{array}{l}\text { Revista Latinoamericana de } \\
\text { Hipertensión }\end{array}$ & $\begin{array}{l}\text { http://www2.scielo.org.ve/scielo.php/script_sci_serial/ } \\
\text { pid_1856-4550/lng_es/nrm_iso }\end{array}$ \\
\hline $\mathrm{AP}$ & Atención Primaria & $\begin{array}{l}\text { http://www.semfyc.es/es/informativo/ } \\
\text { revista+Atenci\%F3n+Primaria/ }\end{array}$ \\
\hline GS & Gaceta Sanitaria & $\begin{array}{l}\text { http://scielo.isciii.es/scielo.php/script_sci_serial/ } \\
\text { pid_0213-9111/lng_en/nrm_iso }\end{array}$ \\
\hline REN & $\begin{array}{l}\text { Revista Ecuatoriana de } \\
\text { Neurología }\end{array}$ & $\begin{array}{l}\text { http://www.medicosecuador.com/revecuatneurol/ } \\
\text { revistas_anteriores.htm }\end{array}$ \\
\hline RIC & Revista de investigación clínica & $\begin{array}{l}\text { http://www.scielo.org.mx/scielo.php?script=sci_ } \\
\text { serial\&pid=0034-8376 }\end{array}$ \\
\hline $\mathrm{CE}$ & Cirugía Española & $\begin{array}{l}\text { http://www.sciencedirect.com/science/ } \\
\text { journal/0009739X }\end{array}$ \\
\hline CIGO & $\begin{array}{l}\text { Clínica e Investigación en } \\
\text { Ginecología y Obstetricia }\end{array}$ & $\begin{array}{l}\text { http://www.sciencedirect.com/science/ } \\
\text { journal/0210573X }\end{array}$ \\
\hline $\mathrm{CM}$ & Colombia Médica & $\begin{array}{l}\text { http://colombiamedica.univalle.edu.co/indexingles. } \\
\text { html }\end{array}$ \\
\hline NDYT & $\begin{array}{l}\text { Revista de Nefrología, Diálisis } \\
\text { y Trasplante }\end{array}$ & $\begin{array}{l}\text { http://www.scimagojr.com/journalsearch. } \\
\text { php?q=144825\&tip=sid\&clean=0 }\end{array}$ \\
\hline MC & Medicina Clínica & $\begin{array}{l}\text { http://www.sciencedirect.com/science/ } \\
\text { journal/00257753 }\end{array}$ \\
\hline EYN & Endocrinología y Nutrición & $\begin{array}{l}\text { http://www.sciencedirect.com/science/ } \\
\text { journal/15750922 }\end{array}$ \\
\hline ADS & Actas Dermo-Sifiliográficas & $\begin{array}{l}\text { http://www.sciencedirect.com/science/ } \\
\text { journal/00017310 }\end{array}$ \\
\hline REQ & $\begin{array}{l}\text { Revista Española de } \\
\text { Quimioterapia }\end{array}$ & http://recyt.fecyt.es/index.php/REQ \\
\hline CIA & $\begin{array}{l}\text { Clínica e Investigación en } \\
\text { Arteriosclerosis }\end{array}$ & $\begin{array}{l}\text { http://www.sciencedirect.com/science/ } \\
\text { journal/02149168 }\end{array}$ \\
\hline $\mathrm{AB}$ & Archivos Bronconeumológicos & $\begin{array}{l}\text { http://www.sciencedirect.com/science/ } \\
\text { journal/17310435 }\end{array}$ \\
\hline
\end{tabular}




\begin{tabular}{|l|l|l|}
\hline CÓDIGO & REVISTA & DIRECCIÓN ELECTRÓNICA \\
\hline IE & Investigación Económica & $\begin{array}{l}\text { http://www.economia.unam.mx/publicaciones/ } \\
\text { web_invesecon/index.html }\end{array}$ \\
\hline ETE & El Trimestre Económico & $\begin{array}{l}\text { http://dialnet.unirioja.es/servlet/ } \\
\text { revista?codigo=4264 }\end{array}$ \\
\hline REA & Revista de Economía Aplicada & $\begin{array}{l}\text { http://dialnet.unirioja.es/servlet/ } \\
\text { revista?codigo=1155 }\end{array}$ \\
\hline EEEE & Estudios de Economía & $\begin{array}{l}\text { http://redalyc.uaemex.mx/src/inicio/HomRevRed. } \\
\text { jsp?iCveEntRev=221 }\end{array}$ \\
\hline IIEE & Investigaciones Económicas & $\begin{array}{l}\text { http://redalyc.uaemex.mx/src/inicio/HomRevRed. } \\
\text { jsp?iCveEntRev=173 }\end{array}$ \\
\hline
\end{tabular}

\begin{tabular}{|l|l|l|}
\hline CÓDIGO & REVISTA & DIRECCIÓN ELECTRÓNICA \\
\hline EMERITA & $\begin{array}{l}\text { Emerita. Revista de Lingüística y } \\
\text { Filología Clásica }\end{array}$ & $\begin{array}{l}\text { http://emerita.revistas.csic.es/index.php/ } \\
\text { emerita }\end{array}$ \\
\hline RLA & $\begin{array}{l}\text { Revista de Lingüística Teórica y } \\
\text { Aplicada }\end{array}$ & $\begin{array}{l}\text { http://www.scielo.cl/scielo.php?pid=0718- } \\
\text { 4883\&script=sci_serial }\end{array}$ \\
\hline SIGNOS & $\begin{array}{l}\text { Revista Signos. Estudios de } \\
\text { Lingüística }\end{array}$ & $\begin{array}{l}\text { http://www.scielo.cl/scielo.php?script=sci_ } \\
\text { serial\&pid=0718-0934\&lng=es\&nrm=iso }\end{array}$ \\
\hline EEFF & Estudios Filológicos & $\begin{array}{l}\text { http://www.scielo.cl/scielo.php?script=sci_ } \\
\text { serial\&pid=0071-1713\&lng=es\&nrm=iso }\end{array}$ \\
\hline CLAC & $\begin{array}{l}\text { Círculo de lingüística aplicada a la } \\
\text { http://www.ucm.es/info/circulo/ }\end{array}$ \\
\hline
\end{tabular}


Anexo 2: Lista de verbos discursivos encontrados en Corpus MEL-2011.

\begin{tabular}{|c|c|c|}
\hline aclarar & detallar & observar \\
\hline acotar & discutir & plantear \\
\hline admitir & ejemplificar & postular \\
\hline advertir & emplear el término & preguntarse \\
\hline afirmar & enfatizar & profundizar \\
\hline agradecer & enunciar & proponer \\
\hline agregar & esbozar & recalcar \\
\hline aludir & esclarecer & recomendar \\
\hline anotar & especificar & reiterar \\
\hline añadir & explicar & referirse \\
\hline apuntar & exponer & reportar \\
\hline argüir & denominar & resaltar \\
\hline argumentar & formular & reseñar \\
\hline aseverar & fundamentar & revisar \\
\hline bosquejar & hacer hincapié & resumir \\
\hline citar & hacer mención & sostener \\
\hline comentar & hacer notar & señalar \\
\hline conceder & ilustrar & subrayar \\
\hline concluir & indicar & sugerir \\
\hline decir & informar & tratar una cuestión \\
\hline definir & insistir & volver sobre una cuestión \\
\hline dejar constancia & llamar & \\
\hline desarrollar (un argumento) & matizar & \\
\hline describir & mencionar & \\
\hline destacar & notar & \\
\hline
\end{tabular}


Anexo 3: Inventario de marcadores de metatexto encontrados en el Corpus MEL2011.

Reformuladores explicativos (REF): es decir, o sea, esto es, i.e., en otras palabras, a saber, se relaciona con, equivale a decir, corresponde a, quiere decir que, equivale a, esto es equivalente a decir que, se entiende como, que no es otra cosa que, puesto en otros términos, vale decir, mejor dicho, dicho de otra manera / forma, dicho en otros términos, dicho de otro modo, en términos sencillos, en otras palabras, tiene que ver con, está relacionado con, en el sentido de, entendido como, esto quiere decir que, en términos más precisos, esto (no) significa, entiéndase, o mejor dicho, vale decir, por así decirlo, mutatis mutandis, en sentido laxo, ceteris paribus, o lo que es lo mismo, dicho llanamente, o más estrictamente hablando, en pocas palabras, en resumidas palabras, en forma resumida, equivaldría a señalar, entendiendo por ello.

\section{Marcadores endofóricos (FOR):}

Marcadores anafóricos: previo(a), anteriormente, arriba, más arriba, al principio, ya, anterior(es), acaba de, antes, allí, líneas arriba, volviendo a, con anterioridad, precedente(s), al comienzo, recién, antes de, al inicio, supra, en un principio, previamente, más atrás, de entrada, inicialmente, previo(a), esto último, lo anterior, hasta aquí.

Marcadores catafóricos: posteriormente, más abajo, más adelante, siguiente(s), próximo(a), a continuación, líneas abajo, como sigue, lo que sigue, seguidamente, en lo que sigue, en adelante, en lo sucesivo, luego, ulterior, próximo, enseguida, infra, luego, posterior (es), de aquí en adelante, (en) el resto de.

Deícticos: ahora, aquí, he aquí, (el / la) presente.

Otros marcadores endofóricos: a lo largo de, durante todo, en el transcurso de, primero, segundo, tercero, finalmente, antes de, último(a), por último, en primer lugar, en segundo lugar, de momento, de aquí en más, al final, tras, de aquí, primeramente, hacia el final. 